Groups Geom. Dyn. 4 (2010), 835-846

DOI $10.4171 / \mathrm{GGD} / 109$
Groups, Geometry, and Dynamics

(C) European Mathematical Society

\title{
On the extraction of roots in exponential $A$-groups
}

\author{
Stephen Majewicz and Marcos Zyman*
}

\begin{abstract}
An exponential $A$-group is a group which comes equipped with an $A$-action ( $A$ is a commutative ring with unity), satisfying certain axioms. In this paper, we investigate some aspects of root extraction in the category of exponential $A$-groups. Of particular interest is the extraction of roots in nilpotent $R$-powered groups. Among other results, we prove that if $R$ is a PID and $G$ is a nilpotent $R$-powered group for which root extraction is always possible, then the torsion $R$-subgroup of $G$ lies in the center. Furthermore, if the torsion $R$-subgroup is finitely $R$-generated, then $G$ is torsion-free.
\end{abstract}

Mathematics Subject Classification (2010). 20F18, 20F19; 13C12, $13 \mathrm{C} 13$.

Keywords. Nilpotent $R$-powered group, exponential $A$-group, extraction of roots.

\section{Introduction}

Let $G$ be a group, $n$ a positive integer, and $g$ an element of $G$. Then $g$ is said to have an $n^{\text {th }}$ root if there exists $h \in G$ such that $h^{n}=g$. An element of $G$ need not have an $n^{\text {th }}$ root. On the other hand, there may be elements of $G$ with more than one $n^{\text {th }}$ root.

A group $G$ in which every element has an $n^{\text {th }}$ root for every integer $n>0$ is termed a radicable or complete (or divisible when $G$ is abelian) group. Thus, for every $g \in G$ and every integer $n>0$, there exists $h \in G$ such that $g=h^{n}$. One can interpret this definition in terms of mappings: $G$ is radicable if and only if the map

$$
\psi: G \rightarrow G \text { defined by } \psi(g)=g^{n}
$$

is surjective for every $n>0$.

If every element of $G$ has at most one $n^{\text {th }}$ root (that is, $n^{\text {th }}$ roots are unique when they exist), then $G$ is called an $R$-group. Thus, if $g, h \in G$ and $g^{n}=h^{n}$ for some integer $n>0$, then $g=h$. Put another way, $G$ is an $R$-group if and only if the mapping $\psi$ defined above is injective.

In [1], Baumslag developed the theory of certain groups containing radicable groups, $R$-groups, and radicable $R$-groups as special cases. For each non-empty set of primes $\omega$, he defined the following classes:

\footnotetext{
${ }^{*}$ The first-named author was supported by the PSC-CUNY Research Award Program (Grant \# 62302).
} 
- $\mathcal{E}_{\omega}$ denotes the class of groups in which $p^{\text {th }}$ roots exist for all $p \in \omega$;

- $U_{\omega}$ denotes the class of groups in which $p^{\text {th }}$ roots are unique (whenever they exist) for all $p \in \omega$;

- $D_{\omega}$ denotes the class $\mathcal{E}_{\omega} \cap \boldsymbol{U}_{\omega}$.

As before, these notions can be described in terms of maps. If $G$ is a group and $\psi: G \rightarrow G$ is defined as $\psi(g)=g^{p}$ for some prime $p \in \omega$, then $G \in \mathcal{E}_{\omega}$ if and only if $\psi$ is surjective, $G \in \mathcal{U}_{\omega}$ if and only if $\psi$ is injective, and $G \in D_{\omega}$ if and only if $\psi$ is bijective. In case $\omega$ is the set of all primes, then the classes $\varepsilon_{\omega}, u_{\omega}$, and $\mathscr{D}_{\omega}$ are denoted by $\mathcal{E}, \mathcal{U}$, and $\mathscr{D}$, respectively. Thus, in the terminology set forth by Baumslag, a $\mathcal{U}$-group is an $R$-group, an $\mathcal{E}$-group is a radicable group, and a $\mathscr{D}$-group is a radicable $R$-group.

Our research focuses on the study of the classes $\mathcal{E}_{\omega}, U_{\omega}$ and $D_{\omega}$ in the category of exponential $A$-groups, where $A$ is an integral domain and $\omega$ is a non-empty set of primes in $A$. Of particular interest is the category of nilpotent $R$-powered groups, where $R$ is a binomial ring. The results presented in this paper have been selected from a work in progress by the authors and deal mainly with nilpotent $R$-powered groups.

We recall the definition of an exponential $A$-group (see [12]).

Definition. An exponential $A$-group is a group $G$, equipped with an action by a commutative ring with unity $A$, such that for all $g \in G$ and for all $\alpha \in A$, the element $g^{\alpha} \in G$ is uniquely defined and the following axioms hold:

(1) $g^{1}=g, g^{\alpha} g^{\beta}=g^{\alpha+\beta}$, and $\left(g^{\alpha}\right)^{\beta}=g^{\alpha \beta}$ for all $g \in G$ and $\alpha, \beta \in A$.

(2) $\left(h^{-1} g h\right)^{\alpha}=h^{-1} g^{\alpha} h$ for all $g, h \in G$ and $\alpha \in A$.

(3) If $g$ and $h$ are commuting elements of $G$, then $(g h)^{\alpha}=g^{\alpha} h^{\alpha}$ for all $\alpha \in A$.

Examples of exponential $A$-groups include $A$-modules, Lyndon's free $\mathbb{Z}[x]$-group [6] and Baumslag's $\mathbb{Q}$-completion of a free group [1]. Categorical notions such as $A$-subgroup, normal $A$-subgroup, and $A$-homomorphism are defined in the obvious way. The interested reader should consult the works of Majewicz ([8] and [10]), and Myasnikov and Remeslennikov [12] for more details.

In this paper, $A$ will always be an integral domain and $\mathcal{M}_{A}$ will denote the class of exponential $A$-groups.

A rich collection of exponential $A$-groups consists of the nilpotent $R$-powered groups, where $R$ is a binomial ring. Recall that a binomial ring $R$ is an integral domain of characteristic zero with unity such that for any $r \in R$ and $k \in \mathbb{Z}^{+}$,

$$
\left(\begin{array}{l}
r \\
k
\end{array}\right)=\frac{r(r-1) \ldots(r-k+1)}{k !} \in R .
$$

The definition of a nilpotent $R$-powered group is due to Hall (see [3]). 
Definition. Let $G$ be a (locally) nilpotent group, and suppose that $G$ is equipped with an action by a binomial ring $R$, such that for all $g \in G$ and for all $\alpha \in R$, the element $g^{\alpha} \in G$ is uniquely defined. Then $G$ is termed a nilpotent $R$-powered group if the following axioms hold:

(1) $g^{1}=g, g^{\alpha} g^{\beta}=g^{\alpha+\beta}$ and $\left(g^{\alpha}\right)^{\beta}=g^{\alpha \beta}$ for all $g \in G$ and $\alpha, \beta \in R$.

(2) $\left(h^{-1} g h\right)^{\alpha}=h^{-1} g^{\alpha} h$ for all $g, h \in G$ and for all $\alpha \in R$.

(3) If $\left\{g_{1}, \ldots, g_{n}\right\} \subset G$ and $\alpha \in R$, then

$$
g_{1}^{\alpha} \ldots g_{n}^{\alpha}=\tau_{1}(\bar{g})^{\alpha} \tau_{2}(\bar{g})^{\left(\begin{array}{c}
\alpha \\
2
\end{array}\right)} \ldots \tau_{k-1}(\bar{g})^{\left(\begin{array}{c}
\alpha \\
k-1
\end{array}\right)} \tau_{k}(\bar{g})^{\left(\begin{array}{c}
\alpha \\
k
\end{array}\right),},
$$

where $k$ is the class of the nilpotent group generated by $\left\{g_{1}, g_{2}, \ldots, g_{n}\right\}$ and $\bar{g}=\left(g_{1}, \ldots, g_{n}\right)$.

Axiom (3) is called the Hall-Petresco axiom and the $\tau_{i}(\bar{g})$ 's are the Hall-Petresco words. By setting $\alpha=1, \alpha=2$, and so on, one can compute the Hall-Petresco words:

$$
\tau_{1}(\bar{g})=g_{1} \ldots g_{n}, \quad \tau_{2}(\bar{g})=\left(g_{1} \ldots g_{n}\right)^{-2}\left(g_{1}^{2} \ldots g_{n}^{2}\right), \text { etc. }
$$

By a theorem of Hall [3], each $\tau_{i}(\bar{g})$ is contained in $\gamma_{i}(G)$, the $i^{\text {th }}$ term of the lower central series of $G$. This allows one to deduce that a nilpotent $R$-powered group is, indeed, an exponential $R$-group.

A well-known example of a nilpotent $R$-powered group is the Mal'cev completion of a torsion-free locally nilpotent group $G$, which is a torsion-free locally nilpotent radicable group containing $G$ (see [7]). In the terminology set forth, this is just a nilpotent $\mathbb{Q}$-powered group. Other examples of nilpotent $R$-powered groups can be found in the works of Hall [3], Majewicz [9], and Warfield [14].

From this point on, $R$ will always be a binomial ring and $\mathcal{N}_{R}$ will denote the class of nilpotent $R$-powered groups.

In Section 2 we provide some preliminary material on exponential $A$-groups and nilpotent $R$-powered groups.

Section 3 contains a selection of results on $\mathcal{U}_{\omega}$-groups and $\mathcal{E}_{\omega}$-groups in the class $\mathcal{N}_{R}$. We begin by proving

Theorem 3.1. Suppose that $G \in \mathcal{N}_{R}$, where $R$ contains $\mathbb{Q}$. If $\left\{g_{1}, \ldots, g_{m}\right\} \subset G$, then the product $g_{1}^{\beta} \ldots g_{m}^{\beta}$ has a $\beta^{\text {th }}$ root for any $\beta \in R$.

A well-known theorem of Mal'cev [7] states that if $\omega$ is a non-empty set of primes, then every $\omega$-torsion-free nilpotent group is a $\mathcal{U}_{\omega}$-group. Our next theorem generalizes this result for nilpotent $R$-powered groups.

Theorem 3.2. Let $\omega$ be a non-empty set of primes in R. A nilpotent R-powered group $G$ is a $U_{\omega}$-group if and only if it is $\omega$-torsion-free. 
Next we prove a theorem similar to one due to Baumslag [1]. In [8], Majewicz introduced the notion of a $\pi$-primary component of a nilpotent $R$-powered group. If $G \in \mathcal{N}_{R}$ and $\pi \in R$ is prime, then the $\pi$-primary component of $G$ is the set

$$
G_{\pi}=\left\{g \in G \mid g^{\pi^{k}}=1 \text { for some } k \in \mathbb{Z}^{+}\right\} .
$$

Theorem 3.3. If $G \in \mathcal{N}_{R}$ is an $\mathcal{E}_{\omega}$-group for some non-empty set of primes $\omega$ and $\pi \in \omega$, then $G_{\pi}$ is an $R$-subgroup of $Z(G)$, the center of $G$.

A consequence of this theorem which generalizes a result of Černikov (see [2] or [5], p. 234) is

Corollary 3.4. Let $R$ be a PID. If $G \in \mathcal{N}_{R}$ is an $\varepsilon$-group, then $\tau(G)$ is an $R$-subgroup of $Z(G)$, where $\tau(G)$ is the torsion $R$-subgroup of $G$.

Another theorem of Černikov that carries over to nilpotent $R$-powered groups is

Theorem 3.7. If $G \in \mathcal{N}_{R}$ is an $\mathcal{E}$-group and $\tau(G)$ is finitely $R$-generated, then $G$ is torsion-free.

\section{Preliminaries}

In this section we provide some elementary results on exponential $A$-groups and nilpotent $R$-powered groups.

Notation: If $H$ is an $A$-subgroup (a normal $A$-subgroup) of an exponential $A$-group $G$, then we write $H \leq_{A} G\left(H \unlhd_{A} G\right)$.

We begin by stating a useful computational lemma. The proof follows from a result of Hall [3] which states that $\tau_{i}(\bar{g}) \in \gamma_{i}(G)$ for each $i \geq 1$.

Lemma 2.1. Let $G \in \mathcal{N}_{R}$ and $\alpha \in R$. If $g_{1}$ and $g_{2}$ commute in $G$, then

$$
\left(g_{1} g_{2}\right)^{\alpha}=g_{1}^{\alpha} g_{2}^{\alpha}
$$

We remind the reader of some well-known commutator identities.

Lemma 2.2. Let $x, y$ and $z$ be elements of any group. Then

$$
[x y, z]=y^{-1}[x, z] y[y, z] \text { and }[x, y z]=[x, z] z^{-1}[x, y] z .
$$

Using the Hall-Petresco axiom, one can establish the following identity (see [14], p. 86): 
Lemma 2.3. Let $G \in \mathcal{N}_{R}$, and suppose that $[g, h] \in Z(G)$ for some $g, h \in G$. Then for any $\mu \in R$,

$$
\left[g^{\mu}, h\right]=\left[g, h^{\mu}\right]=[g, h]^{\mu} .
$$

Using Lemmas 2.2 and 2.3, one can prove

Lemma 2.4. If $G \in \mathcal{N}_{R}$ is non-abelian, then there exists an $R$-homomorphism from $G$ into $Z(G)$ whose image is non-trivial.

The factor group of an exponential $A$-group by a normal $A$-subgroup need not be an exponential $A$-group (see [10] or [12]).

Definition 2.1. Let $G \in \mathcal{M}_{A}$ and $N \unlhd_{A} G$. We call $N$ an ideal of $G$ if

$$
[g, h] \in N \Longrightarrow h^{-\alpha} g^{-\alpha}(g h)^{\alpha} \in N \quad \text { for any } g, h \in G \text { and } \alpha \in A \text {. }
$$

Lemma 2.5. If $G \in \mathcal{M}_{A}$ and $N$ is an ideal of $G$, then the A-action on $G$ induces an A-action on $G / N$,

$$
(g N)^{\mu}=g^{\mu} N \quad \text { for all } g N \in G / N \text { and } \mu \in A,
$$

which turns $G / N$ into an exponential A-group.

If $G \in \mathcal{N}_{R}$, then an application of the Hall-Petresco axiom shows that every normal $R$-subgroup of $G$ is an ideal. It can readily be verified that the isomorphism theorems hold for nilpotent $R$-powered groups.

Definition 2.2. Let $G \in \mathcal{M}_{A}$, and let $S=\left\{s_{1}, \ldots, s_{j}\right\}$ be a subset of $G$. Then

$$
H=\bigcap_{S \subset H_{i} \leq A_{A}}\left\{H_{i}\right\}=\mathrm{gp}_{A}\left(s_{1}, \ldots, s_{j}\right)
$$

is called the $A$-subgroup of $G$, which is $A$-generated by $s_{1}, \ldots, s_{j}$. We term $S$ a set of A-generators for $H$.

The next theorem can be found in [14], p. 87.

Theorem 2.6. The upper and lower central subgroups of a nilpotent R-powered group $G$, denoted by $\zeta_{i}(G)$ and $\gamma_{i}(G)$ respectively, are $R$-subgroups of $G$.

Recall that if $\left\{g_{1}, \ldots, g_{n}\right\}$ is a set of elements in a group $G$, then

$$
\left[g_{1}, \ldots, g_{n}\right]=\left[\left[g_{1}, \ldots, g_{n-1}\right], g_{n}\right]
$$

is a simple commutator of weight $n$ (see [13], p. 123). 
Lemma 2.7. If $G \in \mathcal{N}_{R}$, then

$$
\gamma_{n}(G)=\operatorname{gp}\left(\left[g_{1}, \ldots, g_{n}\right] \mid g_{i} \in G\right) .
$$

The next lemma is useful for proving nilpotency by induction on the class.

Lemma 2.8. If $G \in \mathcal{N}_{R}$ is of class $c \geq 2$ and $g \in G$, then $H=\operatorname{gp}_{R}\left(g, \gamma_{2}(G)\right)$ is of class at most $c-1$.

It is well known that subgroups of finitely generated nilpotent groups are finitely generated. In the case of finitely $R$-generated nilpotent $R$-powered groups, this property is inherited by $R$-subgroups provided that $R$ is a certain type of ring. The next theorem is mentioned in [4].

Theorem 2.9. If $R$ is a noetherian ring and $G$ is a finitely $R$-generated nilpotent $R$-powered group, then every $R$-subgroup of $G$ is finitely $R$-generated.

The notion of a torsion element in an exponential $A$-group is defined in the obvious way.

Definition 2.3. If $G \in \mathcal{M}_{A}$, then an element $g \in G$ is called a torsion element if there exists a non-zero element $\alpha \in A$ for which $g^{\alpha}=1$. The set of torsion elements of $G$ is denoted by $\tau(G)$. We call $G$ a torsion A-group if $\tau(G)=G$, and a torsion-free A-group if $\tau(G)=1$.

In [14], p. 87, Warfield proves the next theorem which does not hold for exponential $A$-groups in general.

Theorem 2.10. If $G \in \mathcal{N}_{R}$, then $\tau(G) \unlhd_{R} G$ and $G / \tau(G)$ is torsion-free.

From this point on, if $G \in \mathcal{N}_{R}$, then we refer to $\tau(G)$ as the torsion $R$-subgroup of $G$.

In the remainder of this paper, $\omega$ will always denotes a non-empty set of primes in $A$ and $\omega^{\prime}$ will denote the set of all primes in $A$ which are not in $\omega$.

Definition 2.4. An element $\alpha \in A$ is an $\omega$-member if either $\alpha=1$ or all prime divisors of $\alpha$ are in $\omega$. If $G \in \mathcal{M}_{A}$, then an element $g \in G$ is an $\omega$-torsion element if $g^{\alpha}=1$ for some $\omega$-member $\alpha$. If every element of $G$ is an $\omega$-torsion element, we say that $G$ is an $\omega$-torsion group. If the only $\omega$-torsion element of $G$ is the identity, then $G$ is $\omega$-torsion-free.

In case $\omega=\{\pi\}$ for a prime $\pi \in A$, we use the terms $\pi$-torsion and $\pi$-torsion-free.

Theorem 2.11. If $G \in \mathcal{N}_{R}$ and $Z(G)$ is $\omega$-torsion-free, then each factor $R$-group $\zeta_{i+1}(G) / \zeta_{i}(G)$ is $\omega$-torsion-free. Consequently, $G$ is $\omega$-torsion-free. 
The theorem follows from Lemma 2.3 and induction on $i$.

Corollary 2.12. If $G \in \mathcal{N}_{R}$ is $\omega$-torsion-free, then $G / Z(G)$ is $\omega$-torsion-free.

Next we provide the definition of a nilpotent $R$-powered group of finite type introduced by Majewicz in [9].

Definition 2.5. A nilpotent $R$-powered group is of finite type if it is a finitely $R$-generated torsion $R$-group.

By Theorem 2.9, if $R$ is a noetherian ring and $G$ is a finitely $R$-generated nilpotent $R$-powered group, then $\tau(G)$ is of finite type.

One can understand nilpotent $R$-powered groups of finite type by examining their $\pi$-primary components.

Definition 2.6. Let $G \in \mathcal{N}_{R}$ and let $\pi \in R$ be prime. The $\pi$-primary component of $G$ is the set

$$
G_{\pi}=\left\{g \in G \mid g^{\pi^{k}}=1 \text { for some } k \in \mathbb{Z}^{+}\right\} .
$$

If $G=G_{\pi}$, then $G$ is referred to as a $\pi$-primary $R$-group. A finitely $R$-generated $\pi$-primary $R$-group is said to be of finite $\pi$-type.

Nilpotent $R$-powered groups of finite $\pi$-type are the analogues of $p$-groups in the category of finite groups.

The following theorem is due to Majewicz and Zyman [11]:

Theorem 2.13. Let $R$ be a noetherian ring and $\pi$ a prime in $R$. Consider the short exact sequence

$$
1 \rightarrow H \rightarrow G \rightarrow G / H \rightarrow 1
$$

in the category of nilpotent $R$-powered groups. Then $G$ is of finite type (finite $\pi$-type) if and only if $H$ and $G / H$ are both of finite type (finite $\pi$-type).

Consequently, every $R$-subgroup of a nilpotent $R$-powered group of finite $\pi$-type is again of finite $\pi$-type when $R$ is noetherian.

The next result can be proven in a similar way to Theorem 3.25 in [14].

Theorem 2.14. If $G \in \mathcal{N}_{R}$ and $\pi \in R$ is prime, then $G_{\pi} \unlhd_{R} G$.

The direct product of nilpotent $R$-powered groups whose classes are bounded can be turned into a nilpotent $R$-powered group in the obvious way.

In [9], Majewicz proved the following:

Theorem 2.15. Suppose that $R$ is a PID and $G$ is a torsion $R$-group. If $\left\{\pi_{i} \mid i \in I\right\}$ is the set of all primes in $R$, then $G$ is the direct product of the $G_{\pi_{i}}$. 


\section{3. $\mathcal{U}_{\omega}$-groups and $\mathcal{E}_{\omega}$-groups in the class $\mathcal{N}_{R}$}

The first theorem is proven in [9] for the ring $\mathbb{Q}[x]$. For completeness, we recreate the proof for any binomial ring containing $\mathbb{Q}$.

Theorem 3.1. Suppose that $R$ contains $\mathbb{Q}$, and let $G \in \mathcal{N}_{R}$ be of class $c$. Let $\beta$ be any element of $R$. If $g_{1}, \ldots, g_{m} \in G$, then there exists $h \in G$ such that $g_{1}^{\beta} \ldots g_{m}^{\beta}=h^{\beta}$.

Thus, every element of the form $g_{1}^{\beta} \ldots g_{m}^{\beta}$ in $G \in \mathcal{N}_{R}$ has a $\beta^{\text {th }}$ root whenever $R$ contains $\mathbb{Q}$.

Proof. The proof is by induction on $c$. If $c=1$, then $h=g_{1} \ldots g_{m}$ satisfies the theorem by Lemma 2.1 .

Suppose that $c>1$ and assume that the result holds for every nilpotent $R$-powered group of class less than $c$. Suppose that $\operatorname{gp}\left(g_{1}, \ldots, g_{m}\right)$ is of class $k \leq c$. By the Hall-Petresco axiom,

$$
\begin{aligned}
g_{1}^{\beta} \ldots g_{m}^{\beta} & =\tau_{1}(\bar{g})^{\beta} \tau_{2}(\bar{g})^{\left(\begin{array}{c}
\beta \\
2
\end{array}\right)} \ldots \tau_{k-1}(\bar{g})^{\left(\begin{array}{c}
\beta \\
k-1
\end{array}\right)} \tau_{k}(\bar{g})^{\left(\begin{array}{c}
\beta \\
k
\end{array}\right)} \\
& =\tau_{1}(\bar{g})^{\beta}\left[\tau_{2}(\bar{g})^{j_{2}}\right]^{\beta} \ldots\left[\tau_{k-1}(\bar{g})^{j_{k-1}}\right]^{\beta}\left[\tau_{k}(\bar{g})^{j_{k}}\right]^{\beta},
\end{aligned}
$$

where

$$
j_{i}=\frac{(\beta-1)(\beta-2) \ldots(\beta-i+1)}{i !} \text { for } 2 \leq i \leq k .
$$

By the comment preceding Lemma 2.1, $\tau_{i}(\bar{g}) \in \gamma_{2}(G)$ for each $i=2, \ldots, k$. Consequently, each $\tau_{1}(\bar{g}), \tau_{2}(\bar{g})^{j_{2}}, \ldots, \tau_{k}(\bar{g})^{j_{k}}$ is contained in $\operatorname{gp}_{R}\left(\tau_{1}(\bar{g}), \gamma_{2}(G)\right)$ which, by Lemma 2.8, is an $R$-subgroup of $G$ of class less than $c$. By induction,

$$
\tau_{1}(\bar{g})^{\beta}\left[\tau_{2}(\bar{g})^{j_{2}}\right]^{\beta} \ldots\left[\tau_{k-1}(\bar{g})^{j_{k-1}}\right]^{\beta}\left[\tau_{k}(\bar{g})^{j_{k}}\right]^{\beta}=h^{\beta}
$$

for some $h \in \mathrm{gp}_{R}\left(\tau_{1}(\bar{g}), \gamma_{2}(G)\right)$. Therefore, $g_{1}^{\beta} \ldots g_{m}^{\beta}=h^{\beta}$.

A fundamental result of Mal'cev in the theory of nilpotent groups is that every torsion-free nilpotent group admits unique root extraction whenever roots exist (see [7]). This result carries over to nilpotent $R$-powered groups.

Theorem 3.2. A nilpotent $R$-powered group $G$ is a $U_{\omega}$-group if and only if it is $\omega$-torsion-free.

Proof. Suppose that $G$ is an $\omega$-torsion-free group of class $c$. We prove that $G$ is a $u_{\omega}$-group by induction on $c$. If $c=1$, there is nothing to prove.

Let $c>1$, and suppose $g, h \in G$ such that $g^{\pi}=h^{\pi}$ for some $\pi \in \omega$. By Corollary $2.12, G / Z(G)$ is $\omega$-torsion-free. Hence, by induction, there exists an element $z \in Z(G)$ such that $g=h z$. Lemma 2.1 yields

$$
g^{\pi}=(h z)^{\pi}=h^{\pi} z^{\pi}=g^{\pi} z^{\pi} .
$$


Consequently, $z^{\pi}=1$. Since $G$ is $\omega$-torsion-free, $z=1$; that is, $g=h$.

Conversely, if $G$ is a $U_{\omega}$-group and $g \in G$, then $g^{\pi}=1=1^{\pi}$ for some $\pi \in \omega$ implies $g=1$. Thus, $G$ is $\omega$-torsion-free.

We remark that every exponential $A$-group which is a $U_{\omega}$-group is $\omega$-torsion-free, but not every $\omega$-torsion-free exponential $A$-group is a $U_{\omega}$-group.

In [2], Cernikov proved that the torsion elements of a complete $Z A$-group $G$ lie in $Z(G)$ (see [5], p. 234). A similar result holds for nilpotent $R$-powered groups in the class $\mathcal{E}$. We establish this by first proving a generalization of a theorem of Baumslag [1].

Theorem 3.3. If $G \in \mathcal{N}_{R}$ is an $\varepsilon_{\omega}$-group of class c and $\pi \in \omega$, then $G_{\pi} \leq{ }_{R} Z(G)$.

Proof. The bulk of the proof rests on proving that $G / Z(G) \in U_{\omega}$ or equivalently, by Theorem 3.2, that $G / Z(G)$ is $\omega$-torsion-free. Let $g \in G, g \neq 1$, and let $\pi \in \omega$ such that $g^{\pi} \in Z(G)$. There exists an integer $k, 0 \leq k<c$, such that $g \notin \zeta_{k}(G)$ and $g \in \zeta_{k+1}(G)$. We claim that $k=0$; that is, $g \in Z(G)$.

Let $h$ be any element of $G$. If $k=0$, we are done. Assume $k=1$, and suppose $h_{0} \in G$ is a $\pi^{\text {th }}$ root of $h$. Then $[g, h] \in Z(G)$ because $g \in \zeta_{2}(G)$. Thus, by Lemma 2.3,

$$
[g, h]=\left[g, h_{0}^{\pi}\right]=\left[g^{\pi}, h_{0}\right]=1 .
$$

Therefore, $g \in Z(G)$, a contradiction. Hence $k$ cannot be 1 .

Suppose that $k>1$, and assume that the set

$$
S=\left\{\tilde{g} \in \zeta_{i}(G) \mid \tilde{g}^{\pi^{n}} \in Z(G) \text { for some } n \in \mathbb{Z}^{+}\right\}
$$

is contained in $Z(G)$ for $1<i \leq k$. Notice that $g^{\pi} \in Z(G)$ implies both $\left(g^{-1} Z(G)\right)^{\pi}=Z(G)$ and $\left(h^{-1} g h \bar{Z}(G)\right)^{\pi}=Z(G)$ in $G / Z(G)$. Hence, by Theorem 2.14, $[g, h]^{\pi^{m}} Z(G)=Z(G)$ in $G / Z(G)$ for some integer $m \geq 0$; that is, $[g, h]^{\pi^{m}} \in Z(G)$. Since $g \in \zeta_{k+1}(G),[g, h] \in \zeta_{k}(G)$ and so $[g, h] \in S$. Hence, $[g, h] \in Z(G)$. Since this holds for all $h \in G$, we have $g \in \zeta_{2}(G)$, which implies $g \in Z(G)$ as before. This contradicts the assumption that $g \notin \zeta_{k}(G)$ and $k>1$. We conclude that $k \ngtr 1$, so we must have $k=0$, as claimed.

We have established that $g^{\pi} \in Z(G)$ implies $g \in Z(G)$. To complete the proof, observe that if $g \in G_{\pi}$, then there exists an integer $t \geq 0$ such that $g^{\pi^{t}}=1$. Therefore, $g^{\pi^{t}} \in Z(G)$ and, consequently, $g \in Z(G)$.

Our analogue of Černikov's result is:

Corollary 3.4. If $R$ is a PID and $G \in \mathcal{N}_{R}$ is an $\mathcal{E}$-group, then $\tau(G) \leq_{R} Z(G)$.

Proof. This follows from Theorems 2.15 and 3.3. 
Next we prove that if $R$ is a PID and a nilpotent $R$-powered group in $\varepsilon$ has a finitely $R$-generated torsion $R$-subgroup, then the torsion $R$-subgroup must be trivial. This generalizes another result due to Cernikov (see [5], p. 235). First we mention an easy generalization of a well-known fact about abelian groups.

Lemma 3.5. If $R$ is a PID and $G$ is a non-trivial divisible abelian $R$-group, then $G$ is not finitely $R$-generated.

Another useful result is

Lemma 3.6. If $G \in \mathcal{N}_{R}$ is an $\mathcal{E}$-group, then any $R$-homomorphic image of $G$ is also an E-group.

Theorem 3.7. Let $R$ be a PID, and suppose $G \in \mathcal{N}_{R} \cap \mathcal{E}$. If $\tau(G)$ is finitely $R$-generated, then $\tau(G)=1$.

The proof of the theorem further shows that $G$ has an ascending central $R$-series, all of whose factors are divisible abelian torsion-free $R$-groups.

Proof. Suppose that $G$ is a divisible abelian $R$-group and $\tau(G)$ is finitely $R$-generated, and assume $\tau(G) \neq 1$. Let $g \in \tau(G), g \neq 1$, satisfy $g^{\alpha}=1$ for some $\alpha \in R$. If $\mu \in R$, then there exists $h \in G$ such that $g=h^{\mu}$. Since $h^{\mu \alpha}=\left(h^{\mu}\right)^{\alpha}=g^{\alpha}=1$, it follows that $h \in \tau(G)$. Thus, $\tau(G)$ is divisible, contradicting Lemma 3.5.

Next let $G \in \mathcal{E}$ be a non-abelian nilpotent $R$-powered group and suppose that $\tau(G)$ is finitely $R$-generated. We claim that $G$ has a strictly ascending central $R$-series

$$
H_{1}<H_{2}<\cdots<H_{i}<\cdots
$$

satisfying

(1) $H_{i} \cap \tau(G)=1$;

(2) $\tau\left(G / H_{i}\right)=\tau(G) H_{i} / H_{i}$ and is finitely $R$-generated;

(3) $H_{i+1} / H_{i}$ is a divisible torsion-free abelian $R$-group.

To begin, we show that $H_{1}$ exists and satisfies (1) and (2). By Lemmas 2.4 and 3.6, there exist non-trivial $R$-homomorphic images of $G$ in $Z(G)$ which are $\mathcal{E}$-groups. Let $H_{1} \in \mathcal{E}$ be one such $R$-subgroup of $Z(G)$. Then $H_{1}$ is abelian and $\tau\left(H_{1}\right)<_{R} G$ is finitely $R$-generated by Theorem 2.9 . Hence,

$$
H_{1} \cap \tau(G)=\tau\left(H_{1}\right)=1
$$

and (1) holds. Next we prove that $\tau\left(G / H_{1}\right)=\tau(G) H_{1} / H_{1}$ and is finitely $R$ generated, establishing (2). Observe that if $g H_{1} \in \tau\left(G / H_{1}\right)$, then there exists $\alpha \in R$ such that $\left(g H_{1}\right)^{\alpha}=H_{1}$; that is, $g^{\alpha} \in H_{1}$. Since $H_{1} \in \mathcal{E}$, there exists $k \in H_{1}$ such that $g^{\alpha}=k^{\alpha}$. By Lemma 2.1, $\left(g k^{-1}\right)^{\alpha} g^{\alpha} k^{-\alpha}=1$ because $k \in Z(G)$. Thus, 
$g k^{-1} \in \tau(G)$ and $g H_{1}=\left(g k^{-1}\right) H_{1} \in \tau(G) H_{1} / H_{1}$. Now, by the $R$-isomorphism theorems,

$$
\tau(G) H_{1} / H_{1} \cong R \tau(G) /\left(\tau(G) \cap H_{1}\right)=\tau(G) .
$$

Since $\tau(G)$ is finitely $R$-generated by hypothesis, so is $\tau\left(G / H_{1}\right)$.

Next we concoct an $R$-subgroup $H_{k}$, assuming that $H_{k-1}$ has been constructed and satisfies (1)-(3). Notice that $G / H_{k-1} \in \mathcal{E}$ by Lemma 3.6 and $\tau\left(G / H_{k-1}\right)$ is finitely $R$-generated by Theorem 2.9. By Lemmas 2.4 and 3.6, there exists a nontrivial normal $R$-subgroup $H_{k} / H_{k-1}$ of $Z\left(G / H_{k-1}\right)$ which is an $\mathcal{E}$-group. Using the same argument as before and the fact that $\tau\left(G / H_{k-1}\right)=\tau(G) H_{k-1} / H_{k-1}$, we have

$$
\left(\tau(G) H_{k-1} / H_{k-1}\right) \cap\left(H_{k} / H_{k-1}\right)=H_{k-1} .
$$

Thus,

$$
\left(\tau(G) H_{k-1}\right) \cap H_{k}=H_{k-1} .
$$

Since $H_{k-1} \cap \tau(G)=1$, we also have $H_{k} \cap \tau(G)=1$. Moreover,

$$
\tau\left(\left(G / H_{k-1}\right) /\left(H_{k} / H_{k-1}\right)\right)=\left(\tau\left(G / H_{k-1}\right) \cdot\left(H_{k} / H_{k-1}\right)\right) /\left(H_{k} / H_{k-1}\right),
$$

from which it follows that $\tau\left(G / H_{k}\right)=\tau(G) H_{k} / H_{k}$. Hence, $H_{k}$ satisfies the required properties.

The ascending central series $\left\{H_{k}\right\}$ must reach $G$ in a finite numbers of steps since $G$ is nilpotent. Thus, there exists $n>0$ such that $H_{n}=G$. Consequently, $G \cap \tau(G)=1$; that is, $\tau(G)=1$.

\section{References}

[1] G. Baumslag, Some aspects of groups with unique roots. Acta Math. 104 (1960), 217-303. Zbl 178.34901 MR 0122859

[2] S. N. C̆ernikov, Complete groups possessing ascending central series. Mat. Sb. 18 (60) (1946), 397-422. Zbl 0063.07321 MR 0018646

[3] P. Hall, Nilpotent groups. Queen Mary College Mathematics Notes, Mathematics Department, Queen Mary College, London 1969. Zbl 0211.34201 MR 0283083

[4] M. I. Kargapolov, V. N. Remeslennikov, N. S. Romanovskii, V. A. Roman'kov, and V. A. Churkin, Algorithmic problems for $\sigma$-power groups. Algebra i Logika 8 (1969), 643-659; English transl. Algebra and Logic 8 (1969), 364-373. Zbl 0238.20050 MR 0283060

[5] A. G. Kurosh, The theory of groups. Vol. II, Chelsea Publishing, New York 1960. Zbl 0064.25104 MR 109842

[6] R. C. Lyndon, Groups with parametric exponents. Trans. Amer. Math. Soc. 96 (1960), 518-533. Zbl 0108.02501 MR 0151502

[7] A. I. Mal'cev, Nilpotent torsion-free groups. Izv. Akad. Nauk SSSR. Ser. Mat. 13 (1949), 201-212. Zbl 0034.01702 MR 0028843 
[8] S. Majewicz, Nilpotent $\mathbb{Q}[x]$-powered groups and $\mathbb{Z}[x]$-groups. Ph.D. thesis, The City University of New York, New York 2004.

[9] S. Majewicz, Nilpotent $\mathbb{Q}[x]$-powered groups. In Combinatorial group theory, discrete groups, and number theory, Contemp. Math. 421, Amer. Math. Soc., Providence, RI, 2006, 227-249. Zbl 1173.20313 MR 2303839

[10] S. Majewicz, On classes of exponential A-groups. Comm. Algebra 38 (2010), 1363-1384. Zbl 05710465 MR 2656582

[11] S. Majewicz and M. Zyman, Power-commutative nilpotent $R$-powered groups. Groups Complex. Cryptol. 1 (2009), 297-309. Zbl 05680262 MR 2598996

[12] A. G. Myasnikov and V. N. Remeslennikov, Groups with exponents I. Fundamentals of the theory and tensor completions. Sibirsk. Mat. Zh. 35 (1994), 1106-1118; English transl. Siberian Math. J. 35 (1994), 986-996. Zbl 0851.20050 MR 1308240

[13] D. J. S. Robinson, A course in the theory of groups. 2nd ed., Graduate Texts in Math. 80, Springer-Verlag, New York 1996. Zbl 0836.20001 MR 1357169

[14] R. B. Warfield, Jr., Nilpotent groups. Lecture Notes in Math. 513, Springer-Verlag, Berlin 1976. Zbl 0347.20018 MR 0409661

Received September 28, 2009; revised March 24, 2010

S. Majewicz, Department of Mathematics, Kingsborough Community College, 2001 Oriental Boulevard, Brooklyn, NY 11235-2398, U.S.A.

E-mail: smajewicz@kbcc.cuny.edu

M. Zyman, Department of Mathematics, Borough of Manhattan Community College, 199 Chambers Street, N-520, New York, NY 10007, U.S.A.

E-mail: mzyman@bmcc.cuny.edu 